\title{
Anomalous transfer in rats: Pattern element separation and discriminability
}

\author{
P. C. DODWELL, H. B. FERGUSON, and R. R. NIEMI \\ Queen's University, Kingston, Ontario, Canada
}

\begin{abstract}
Anomalous transfer (AT) means anomalous preference on a transfer test, following conventional discrimination learning. It occurs when a subject chooses a novel stimulus over the training-positive stimulus, or prefers the training-negative stimulus to a novel one. AT has been demonstrated in several small mammals, but only with one stimulus set. Animals trained with patterns of small squares arranged in rows or columns subsequently find stripes in the appropriate orientation "more positive" or "more negative" than the respective training patterns. In the present experiments, it is shown that AT does not depend on particular values for the parameters of the training and transfer shapes, such as relative degrees of horizontal and vertical separation among pattern elements. These findings support the hypothesis that AT is a transpositional discrimination phenomenon that can be explained in terms of the contour coding system of mammalian vision.
\end{abstract}

Anomalous transfer (AT) is defined as a choice made on a transfer trial, following conventional discrimination training to positive and negative stimuli, in which a novel stimulus is preferred to the training-positive discriminandum or the trainingnegative stimulus is preferred to a novel one. That is, AT occurs when the training contingencies are directly contradicted in subsequent transfer trials. It appears to have been first discovered by Krechevsky (1938), who found that rats trained to discriminate between the row and column patterns of Figure 1 (above) would subsequently prefer smooth vertical stripes to columns, as shown in Figure 1 (below), if they had been trained with rows positive. Krechevsky interpreted this finding in terms of the then-current notions of Gestalt psychology. Dodwell (1970) confirmed the finding and also demonstrated that AT occurs to the training-negative pattern. That is, for example, animals trained with columns negative would subsequently prefer columns to vertical stripes; the latter were "more negative" than the training-negative pattern. It was also demonstrated that AT is a genuine effect of prior discrimination learning and cannot be accounted for in terms of initial preferences in the experimental subjects or responses to novel patterns. Anomalous transfer has been demonstrated in rats and squirrels (Dodwell, 1970) and in squirrel monkeys (Dodwell, Litner, \& Niemi, 1970) using the shapes shown in Figure 1. The phenomenon is analogous to peak shift (Terrace, 1966) and to the responses to "supernormal" stimuli studied by some ethologists (e.g., Tinbergen, 1951) but is not identical to either of them. Dodwell (1970)

This research was supported by Grant AOA44 from the National Research Council of Canada to the first author. H. B. Ferguson is now at Carleton University, Ottawa, Ontario. R. R. Niemi is now at the University of Manitoba, Winnipeg, Manitoba.
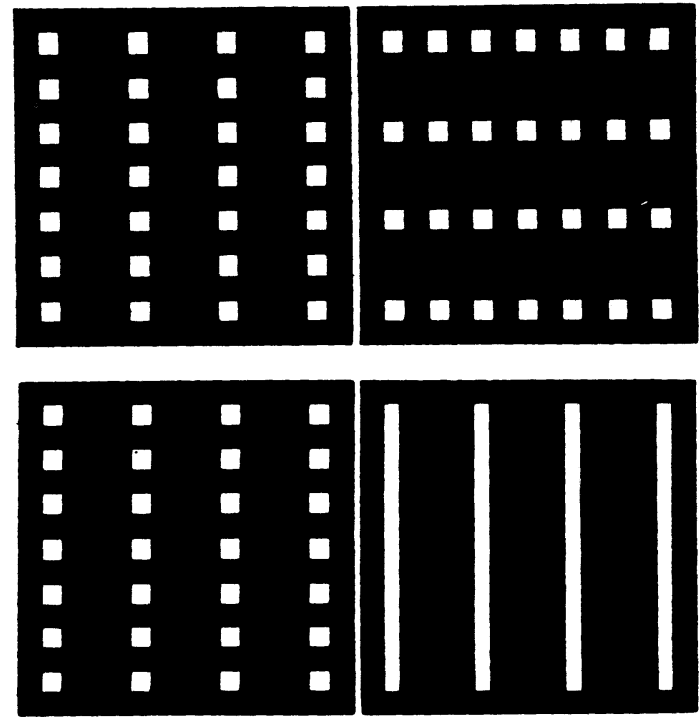

Figure 1. Training stimuli (above) and transfer stimuli (below) used in demonstrating anomalous transfer. After training on the pair above with columns positive, transfers using the pair below show that animals prefer stripes to the training-positive columns.

explained these particular anomalies in terms of a shape coding system which was based on responses in neurons with simple receptive fields of the type described by Hubel and Wiesel in the cat and monkey (Hubel \& Wiesel, 1962, 1968), the idea being that solid stripes would excite such receptive fields more strongly than would rows and columns of interrupted stripes. In that case, the anomalies could be considered instances of transposition along a continuum coded as the outputs from populations of units with horizontally and vertically oriented receptive fields.

Whatever the explanation, the AT phenomenon is robust, having been demonstrated in three species of 
small mammals and with several sorts of apparatus and training method. However, so far it has only been demonstrated with one set of stimulus arrays. The present paper looks at the effect of changing pattern element separation in displays like Figure 1 (above) on their discriminability, and, in doing so, attempts to find stronger evidence for the continuum hypothesis.

\section{EXPERIMENT I}

The object of the first experiment was to examine the limits of discriminability on the horizontalityverticality dimension by using transfer patterns in which the relative separation of horizontal and vertical components is reduced. Original training was done using the patterns of Figure 1 (above).

\footnotetext{
Method

Subjects and apparatus. Ten male Sprague-Dawley hooded rats were used, about 3 months old at the start of training. The apparatus was a discrimination chamber described in detail elsewhere (Niemi \& Dodwell, 1971). It consisted of a chamber $76 \mathrm{~cm}$ long and $15 \mathrm{~cm}$ wide, with a lever at one end and a pair of panels at the other, on which the discriminanda were displayed. Pressing the lever turned on the panel displays and thus initiated a trial. Pressing one of the panels resulted in the presentation of reward or a time-out of $15 \mathrm{sec}$. Programming, timing, and recording were automatically controlled.

Stimuli. The training stimuli were rows and columns of white squares on a black background, as shown in Figure 1(above). The ratio of center-to-center distances between squares is $3: 1$ in these stimuli. The transfer stimuli were similar arrays of rows and columns, but with center-to-center ratios of $2: 1,1.6: 1$, and 1.3:1, the latter being still distinguishable to the human eye as rows and columns, but of course with far less salience than is found in the $3: 1$ arrays.

Procedure. The subjects were brought to $85 \%$ of normal body weight and maintained at that level throughout the experiment. They were trained in the apparatus, as described by Niemi and Dodwell (1971) to choose a lighted panel, using a "noncorrection" procedure. They were then divided randomly into two groups of five, one group trained with rows positive, the other with columns positive, for 50 trials per day to a criterion of $90 \%$ correct on one day. Both groups were then given transfer tests, consisting of 20 trials with the (unreinforced) transfer pairs interspersed with $\mathbf{4 5}$ (reinforced) trials on the original stimuli. There were three different transfer tests, given on separate days, with progressively smaller center-to-center ratios. Order of tests was not balanced as the object was to look for the limit of discriminability. If a rat failed to maintain $90 \%$ on the training stimuli, it was retrained to criterion before transfer testing proceeded. Subsequently all subjects were tested for AT by being presented with rows and horizontal stripes. or columns and vertical stripes.
}

\section{Results and Discussion}

All subjects reached criterion on the training patterns within six sessions ( 300 trials), the quickest doing so in three. All showed transfer to the unfamiliar patterns with closer ratios, which was almost perfect in the case of the 2:1 ratio. Mean preference for the training orientation in this case was $87 \%$ (range: $78-98 \%$ ), for the $1.6: 1$ ratio it was $85.4 \%$ (range: $74-94 \%$ ), and for the $1.3: 1$ ratio it was $74 \%$ (range: $62-92 \%$ ). This suggests that the latter ratio is approaching the limit of discriminability for this sort of pattern in the hooded rat; only one animal performed at better than $80 \%$ correct on this discrimination.

All rats showed AT, i.e., they treated smooth horizontal stripes as more positive than rows, if they had been trained on rows positive, and smooth vertical stripes as more negative than columns. The converse was true for those trained with columns positive. These preferences were at $80 \%$ or better, thus replicating the earlier main finding on AT (Dodwell, 1970).

The results support the notion that rats distinguish between the patterns of figure 1 on a continuum of "horizontality-verticality," and their discriminations are not disrupted by changes along that dimension, even down to pattern ratios which for humans approach the limit of ready discriminability.

\section{EXPERIMENT II}

The object of this experiment was to test the continuum hypothesis by training subjects at one set of ratios, then testing for ease of discrimination, in terms of latency, at others both easier and more difficult. One implication of the continuum hypothesis is that discrimination between the end-points should be easier than discrimination between any intermediate points. This was partially tested by Dodwell (1970), who showed that preference between horizontal vs. vertical stripes was at least as strong as between training patterns of rows and columns at 3:1 ratio, and probably stronger. Ideally, one would hope that latency could be used to measure strength of preference.

\section{Method}

Subjects and apparatus. The subjects were eight male Sprague-Dawley rats. Otherwise conditions were the same as for Experiment I.

Stimuli. The training stimuli were rows and columns at a 2:1 ratio. Transfer pairs were at ratios of 1:1, 1.6:1, 3:1, and also sets of smooth horizontal and vertical stripes.

Procedure. this was the same as for Experiment I up to the transfer testing procedure. For this the subjects were divided into two groups, both given 50 trials per day for 5 days, 1 day on each transfer test, consisting of 40 original training trials among which the 10 transfer trials were randomly interspersed. For Group $A$, the transfer trials were reinforced; for Group B, they were not. The two procedures were introduced in an attempt to find conditions under which latency differences could best be measured. The order of transfer tests was always from "easier" to "more difficult," as predicted by the continuum hypothesis, i.e., the order was from stripes, through the various ratios down to the identical (1:1) pairs.

\section{Results and Discussion}

Group A. Transfer performance was very high on smooth stripes $(99.5 \%)$ and on patterns with ratios of $3: 1$ (mean correct $=98.5 \%$ ), both of which should be easier than the training patterns. However, performance on the 1.6:1 patterns was also excellent 
at $88 \%$ compared to criterion performance of $90 \%$. The transfer to $1: 1$ ratio patterns proved impossible to run for this group, perhaps not surprising in view of the fact that transfer performance consisted of responding to one of a pair of identical patterns which were reinforced on a random basis. After a few trials, the subjects stopped responding to the transfer stimuli. However, this does give a useful control on the possibility that they were responding to other cues such as apparatus noise which might be adventitiously available.

The latency data were entirely idiosyncratic. For example. two rats ran significantly faster to the stripes (easiest) than the 1.6:1 ratio patterns (hardest), $\mathrm{p}<.001$ on individual sign tests, but one showed exactly the reverse, at the same high level of reliability, and one showed no difference.

Group B. Transfer preference with nonreinforced transfer trials was similar to what was found with Group A. Performance on the stripes was $93 \%$ correct, $99 \%$ on the $3: 1$ ratios, and $99 \%$ on the $1.6: 1$. In this case, responses were obtained to the $1: 1$ ratio patterns, one of which was arbitrarily assigned the label "correct." The mean response to it was $51 \%$, thus further ensuring that subjects were making genuine visual discriminations in the other conditions.

The latency data were less bizarre in this case, but again somewhat difficult to interpret. Table 1 shows the mean latency data for the five choice conditions. These are definitely nonrandom: A Friedman one-way analysis of variance yielded $\mathrm{X}_{\mathrm{r}}^{2}=13.6(\mathrm{p}<.01)$. It appears that the stripes, although responded to perfectly, pose a different choice problem than do the rows and columns, and the high latencies of the impossible discriminations are to be expected. However, the data give no ground for hope that latencies will yield a measure for scaling on the horizontality-verticality dimension for this class of pattern. At best, it can be concluded that the
Table 1 Mean Choice Latencies for Patterns Used in Experiment II

\begin{tabular}{crrrrr}
\hline & \multicolumn{5}{c}{ Pattern Ratio } \\
Subjects & $1: 1$ & $1.6: 1$ & $2: 1^{*}$ & $3: 1$ & Stripes \\
\hline $\mathrm{S}_{1}$ & 5.3 & 2.5 & 2.5 & 2.2 & 3.1 \\
$\mathrm{~S}_{2}$ & 30.0 & 3.1 & 3.5 & 3.0 & 6.3 \\
$\mathrm{~S}_{3}$ & 7.0 & 3.0 & 3.9 & 4.0 & 6.7 \\
$\mathrm{~S}_{4}$ & 36.9 & 3.0 & 3.1 & 3.9 & 6.4 \\
Means & 19.8 & 2.9 & 3.75 & 3.28 & 5.63 \\
\hline
\end{tabular}

Note-The patterns were like those shown in Figure 1. Each data point is the mean of 10 latencies.

${ }^{*}$ Training ratio

unreinforced transfer trial procedure is the more appropriate one for obtaining usable latency data. The only solid evidence for the continuum hypothesis, then, comes from the data on discriminatory choice.

\section{REFERENCES}

Dodwell, P. C. Anomalous transfer after pattern discrimination training in rats and squirrels. Journal of Physiological and Comparative Psychology, 1970, 71, 42-51. (a)

Dodwell, P. C., Litner, J. S., \& Niemi, R. R. Anomalous transfer effects following pattern discrimination training in the squirrel monkey. Psychonomic Science, 1970, 18, 5-7. (b)

Hubel, D. H., \& Wiesel, T. N. Receptive fields, binocular interaction and functional architecture in the cat's visual cortex. Journal of Physiology, 1962, 160, 106-154.

Hubel, D. H., \& Wiesel, T. N. Receptive fields and functional architecture of monkey striate cortex. Journal of Physiology, $1968,195,215-234$.

KRECHEVSKY, I. An experimental investigation of the principle of proximity in the visual perception of the rat. Journal of Experimental Psychology, 1938, 22, 497-523.

Niemi, R. R., \& Dodwell, P. C. The salience of two cues for pattern recognition in the rat. Canadian Journal of Psychology, 1971, 25, 375-383.

TERrace, H. S. Stimulus control. In: W. K. Honig (Ed.), Operant behavior. New York: Appleton-Century-Crofts, 1966.

Tinbergen, N. The study of instinct. Oxford: Oxford University Press, 1951.

(Received for publication October 20, 1975.) 